\title{
The Use of Eccentric Strength Training to Enhance Maximal Muscle Strength, Explosive Force (RDF) and Muscular Power - Consequences for Athletic Performance
}

\author{
Per Aagaard*
}

Institute of Sport Science and Clinical Biomechanics, University of Southern Denmark, Odense, Denmark

\begin{abstract}
Heavy-resistance strength training results in marked increases in maximal muscle strength and power in both athletes, untrained subjects and elderly individuals. The addition of eccentric overload training (loads >100\% 1RM) appears to evoke enhanced gains in these parameters. Further, the use of plyometric training evokes marked increases in maximal muscle power. The adaptive physiological mechanisms involve changes in neuromuscular activity and alterations in muscle morphology (fiber size, fiber lengths) and muscle architecture, respectively, while changes in tendon stiffness may potentially contribute as well.
\end{abstract}

Keywords: Neural, eccentric, power, RFD, muscle, hypertrophy, plyometrics.

\section{INTRODUCTION}

Maximal muscle strength and power can be maximized by means of resistance training, however different types of training may lead to differential gains in one or the other parameter. Heavy-resistance strength training (HRST) leads to increased levels of contractile muscle strength during isometric, concentric and eccentric muscle actions of maximal voluntary effort. Corresponding effects of HRST on maximal muscle power production have been reported in the literature, while more specialized types of resistance training (i.e. plyometric exercises) appear to also induce gains in maximal muscle power production.

This brief review article presents and discus the effect of various resistance training modalities, including supramaximal eccentric training and plyometric training, on the maximal force and power production of human skeletal muscle in untrained subjects, athletes and aging individuals.

\section{HEAVY-RESISTANCE STRENGTH TRAINING}

Maximal muscle strength typically is increased by 20 $40 \%$ in response to HRST of medium to moderate time length (8-16 weeks) [1-3]. Slightly greater gains may be seen for maximal muscle power, which typically demonstrates increases of $20-50 \%$ after months of HRST training both in young untrained persons [4-6], elite soccer players [7] (Fig. 1) and old individuals [8]. Notably, HRST appears to evoke similar [4,8] or greater [7] gains in maximum muscle power than low-resistance types of resistance training (cf. Fig. 1).

As a result of adaptive alterations in neural function [911], muscle morphology [3, 12, 13] and architecture [12]

\footnotetext{
*Address correspondence to this author at the Institute of Sport Science and Clinical Biomechanics, University of Southern Denmark, Odense, Denmark, Campusvej 55, DK-5230 Odense M, Denmark; Tel: +45 6550 3448;

Fax: +45 6550 3480; E-mail: paagaard@health.sdu.dk
}

HRST is found to induce greater gains in maximal muscle strength and power compared to low-resistance types of training $[1,4,5,7]$ (cf. Fig. 1). Notably, the increase in maximum muscle strength contributes per se to the gain in maximal muscle power observed following HRST. Furthermore, tendon stiffness may increase with HRST [14, 15], which theoretically allows to perform faster stretchshortening actions (SSC) that for a given muscle work output would translate into an elevated power production.

Excised muscle fibers obtained from subjects exposed to years of resistance training demonstrate greater contractile force and power production compared to that measured in single muscle fibers from untrained age-matched individuals [16] (Fig. 3). Maximum fiber shortening speed as well as muscle fiber power normalized relative to fiber size appear to remain unaffected by HRST [16, 17], altogether suggesting that the superior in vitro power production of single muscle fibers with HRST is mainly the overall result of individual muscle fiber hypertrophy.

\section{ECCENTRIC TRAINING}

Neuromuscular activity is suppressed during maximal eccentric (ECC) muscle contraction in untrained subjects $[10,18]$, due to reduced levels of central activation [19] and reduced efferent motorneuron outflow indicated by diminished evoked V wave responses [20]. Maximum ECC muscle force during MVC can be increased by superimposed electrical muscle stimulation only in untrained individuals and not in trained strength athletes, indicating that the suppression in motorneuron activation may be altered by strength training [21]. In support of this notion, maximum ECC muscle strength was increased by use of HRST [1,2] due to a removed or diminished suppression in neuromuscular activity $[10,22]$. Notably, HRST was found to produce elevated H-reflex and V wave responses during MVC, indicating increased excitability of spinal motorneurons, de- 
creased presynaptic inhibition and elevated descending motor drive $[11,23]$. Notably, the use of supramaximal ECC strength training leads to selectively elevated $\mathrm{V}$ wave responses during maximal eccentric contraction [20], demonstrating that adaptive changes in spinal motorneuron function and/or descending motor drive were evoked during ECC contraction. As a result, maximal ECC muscle strength is markedly increased with this particular type of training (discussed below).

Eccentric resistance training using submaximal, maximal $(100 \% 1 \mathrm{RM})$ or supramaximal (> 100\% 1RM) training loads may lead to greater increases in maximal muscle strength compared to more conventional types of HRST [2, 24-28], in part due to greater or a more pronounced degree of muscle hypertrophy following eccentric training [22, 24-26] (Fig. 2).

The greater gains in maximal muscle strength is expected to result in greater increases in maximal muscle power, since power is constituted by the product between instantaneous muscle force and muscle shortening speed, respectively. Animal experiments have shown that eccentric resistance training, especially when performed at long fiber lengths (i.e. at highly flexed joint positions, such as in deep squats) may lead to sarcomere addition and elongated muscle fiber lengths [29-32]. Notably, elongated muscle fiber lengths will contribute substantially to the gain in maximal muscle power induced by training. Thus, simulation analysis using empirical F-V and P-V relationships obtained for single human muscle fibers reveals that a $10 \%$ increase in maximal isomet- ric muscle fiber force leads to a $10 \%$ gain in maximum (peak) power production, while a $10 \%$ increase in fiber length with a corresponding increase in maximal fiber shortening speed (Vo) cause maximum (peak) power to also increase by $10 \%$. However, the relative gain in muscle power generated at high contraction speed (corresponding to $75 \%$ of $\mathrm{Vo}$ ) is 4-fold higher (42\% vs $10 \%)$ when comparing the effect of increasing muscle fiber length by $10 \% \mathrm{vs}$ increasing maximum isometric force by $10 \%$, respectively. Most potently, however, if a $10 \%$ gain is achieved both for maximum force and muscle fiber length, respectively, this yields a $56 \%$ gain in high-speed power (at $75 \%$ Vo). In comparison, maximum (peak) power production is increased by $21 \%$. Thus, any training induced increase in muscle fiber length is likely to have a strong positive influence on the magnitude of maximal muscle power production, especially during very fast movements.

\section{PLYOMETRIC TRAINING}

Plyometric training involves forceful stretch-shortening (SSC) actions in the muscle-tendon complex [32] that typically involves production of high eccentric muscle forces, and where the magnitude of peak muscle loading (net joint moments) is remarkably similar to those observed during conventional HRST exercises. Consequently, plyometric training may lead to muscle hypertrophy, at least when performed in previously untrained subjects [33, 34], while frequently also leading to more pronounced gains in maximal muscle power than that attained by HRST alone [35]. However, the latter finding may be obscured by the fact that
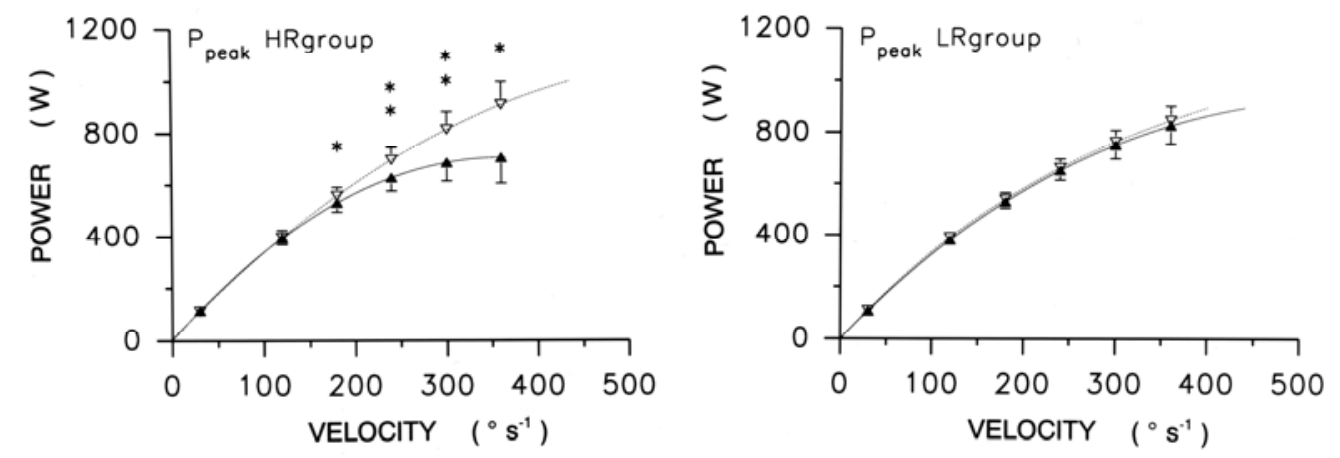

Fig. (1). Peak quadriceps muscle power production before (full line) and after (dotted) 12 wks of heavy-resistance (8 RM, HRgroup) or lowresistance (24 RM, LRgroup) strength training in elite soccer players. Data from Aagaard et al. [7].
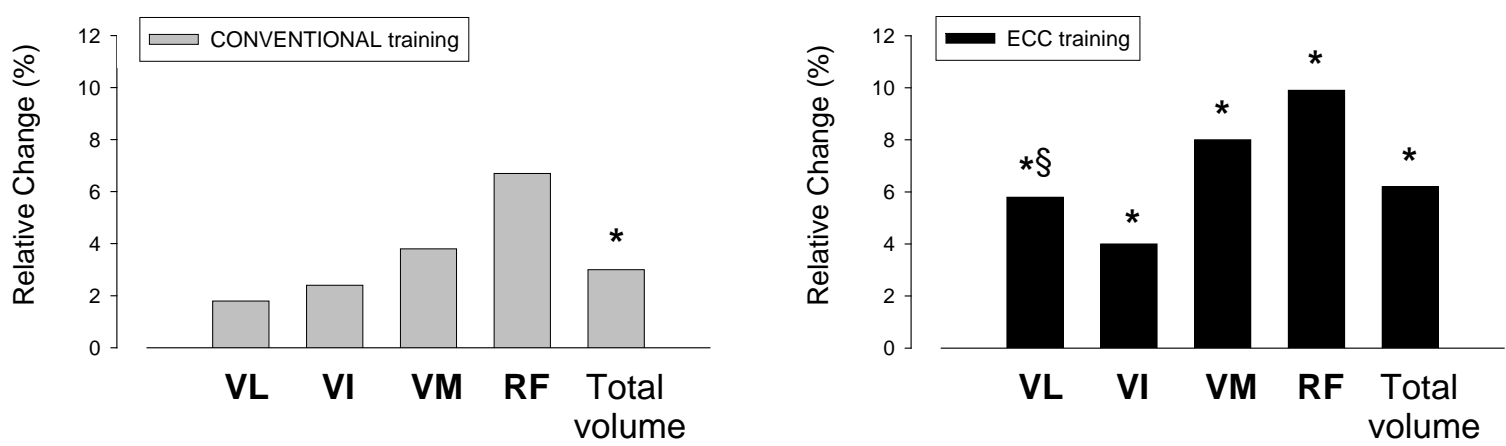

Fig. (2). Changes in quadriceps muscle cross-sectional area after conventional (grey bars) and supramaximal eccentric (black bars) strength training. Data from Norrbrand et al. [27]. 
fibers containing Type I MHC

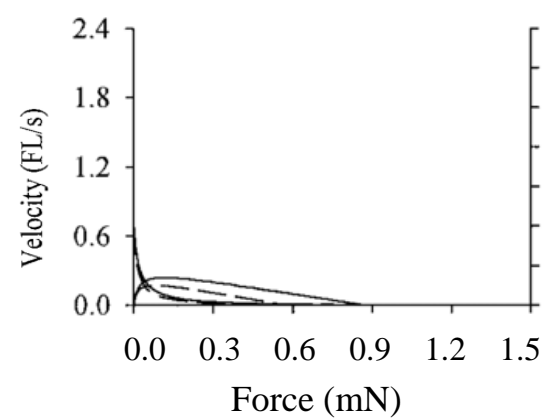

fibers containing Type IIa MHC

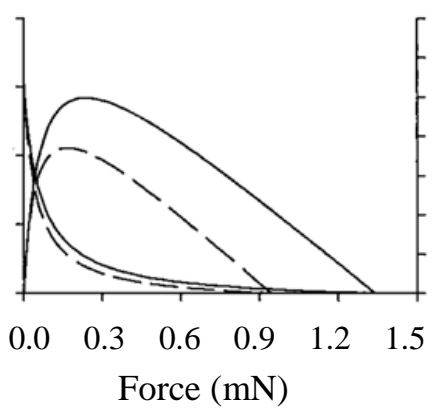

fibers containing Type IIa/IIx MHC

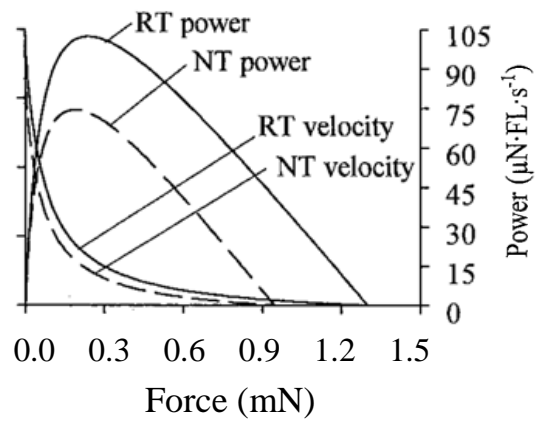

Fig. (3). Force-velocity and power-velocity relationships (group mean curves) obtained for single isolated muscle fibers from long-term resistance trained (RT; $7.6 \pm 1.6$ yrs of RT, full lines) and age matched untrained (NT, dotted lines) individuals. Data from Shoepe et al. [16].

plyometric training typically introduces a large learning effect when subjects are evaluated (tested) in SSC exercises that are identical to those used in the plyometric training. Regardless, for athletes involved in explosive-type events such as sprinting, maximal jumping and throwing, the combination of HRST and plyometric training may lead to enhanced performance and greater gains in maximal muscle power than HRST alone.

Single muscle fiber data obtained in previously untrained subjects (physical education students) before and after plyometric training suggest this training modality results in an elevated maximum muscle fiber shortening speed in vitro, which explains the substantial gain in maximal power production observed for isolated muscle fibers [33].

The optimal loading intensity for maximizing the gain in muscle power with plyometric training remains a subject of debate [35]. In addition, the optimum power load (i.e. the load eliciting maximal power output) varies between muscle groups, and is also affected by the training status of the subject, as well as the choice of exercise [35]. For example, in strength trained individuals maximum leg muscle power was found to occur during unloaded ( $0 \%$ 1RM) squat jumping whereas the optimum load for maximizing the power output during conventional squat exercise was $40-70 \%$ of 1RM [14]. Nevertheless, the optimal training load for inducing maximal longitudinal gains in muscle power may well include very high loading intensities of $80-100 \%$ of 1RM (cf. Fig. 1) to ensure that optimal adaptation take place both in neural function [11, 36] and muscle size [37], and while performed in combination with eccentric training and/or plyometric training exercises. The functional significance of the latter training modality may primarily be to evoke increases in muscle fiber length, and to train ('learn') the CNS to perform very rapid SSC movements.

\section{CONCLUSIONS}

Maximal muscle strength and power are elevated in response to heavy-resistance strength training (HRST), and the addition of eccentric and/or plyometric training appear to evoke enhanced gains in these parameters. The adaptive physiological mechanisms involve changes in neuromuscular activity and alterations in muscle morphology (fiber size, fiber lengths) and muscle architecture, respectively, while changes in tendon stiffness may potentially contribute as well.

\section{REFERENCES}

[1] Aagaard P, Simonsen EB, Trolle M, Bangsbo, J, Klausen K. Specificity of training velocity and training load on gains in isokinetic knee joint strength. Acta Physiol Scand 1996; 156: 123-9.

[2] Colliander EB, Tesch PA. Effects of eccentric and concentric muscle actions in resistance training. Acta Physiol Scand 1990; 140: 31-9.

[3] Häkkinen K, Newton RU, Gordon SE, et al. Changes in muscle morphology, electromyographic activity, and force production characteristics during progressive strength training in young and older men. J Gerontol 1998; 53: B415-23.

[4] Duchateau J, Hainaut K. Isometric or dynamic training: differential effects on mechanical properties of a human muscle. J Appl Physiol 1984; 56: 296-301.

[5] Kaneko M, Fuchimoto T, Toji H. Training effects of different loads on the force-velocity relationship and mechanical power output in human muscle. Scand J Sports Sci 1983; 5: 50-5.

[6] Toji H, Suei, K, Kaneko M. Effects of combined training loads on relations among force, velocity, and power development. Can J Appl Physiol 1997; 22: 328-36.

[7] Aagaard P, Simonsen EB, Trolle M, Bangsbo J, Klausen K. Changes in the human muscle force-velocity relationship in response to resistance training and subsequent detraining. Eur $\mathbf{J}$ Appl Physiol 1994; 69: 382-6.

[8] de Vos NJ, Singh NA, Ross DA, Stavrinos TM, Orr R, Fiatarone Singh MA. Resistance training in older adults. J Gerontol 2005; 60A: 638-47.

[9] Aagaard, P. Training-induced changes in neural function. Exerc Sports Sci Rev 2003; 31: 61-7.

[10] Aagaard P, Simonsen EB, Andersen JL, Magnusson P, HalkjærKristensen J, Dyhre-Poulsen P. Neural inhibition during maximal eccentric and concentric quadriceps contraction: effects of resistance training. J Appl Physiol 2000; 89: 2249-57.

[11] Aagaard P, Simonsen EB, Andersen JL, Magnusson SP, DyhrePoulsen P. Neural adaptation to resistance training: changes in evoked V-wave and H-reflex responses. J Appl Physiol 2002; 92: 2309-318.

[12] Aagaard P, Andersen JL, Dyhre-Poulsen P, et al. A mechanism for increased contractile strength of human pennate muscle in response to strength training: changes in muscle architecture. J Physiol 2001; 534(2): 613-23.

[13] Kraemer WJ, Patton JF, Gordon SE, et al. Compatibility of highintensity strength and endurance training on hormonal and skeletal muscle adaptations. J Appl Physiol 1995; 78: 976-89.

[14] Kongsgaard M, Reitelseder S, Pedersen TG, et al. Region specific patellar tendon hypertrophy in humans following resistance training. Acta Physiol 2007; 191: 111-21.

[15] Reeves ND, Maganaris CN, Narici MV. Effect of strength training on human patella tendon mechanical properties of older individuals. J Physiol 2003; 548: 971-81.

[16] Shoepe TC, Stelzer JE, Garner DP, Widrick JJ. Functional adaptability of muscle fibers to long-term resistance exercise. Med Sci Sports Exerc 2003; 35: 944-51. 
[17] Widrick J, Stelzer J, Shoepe T, Garner D. Functional properties of human muscle fibers after short-term resistance exercise training. Am J Physiol 2002; 283: R408-R16.

[18] Seger JY, Thorstensson A. Muscle strength and myoelectric activity in prepubertal and adult males and females. Eur J Appl Physiol 1994; 69: 81-7.

[19] Babault N, Pousson M, Ballay Y, Van Hoecke J. Activation of human quadriceps femoris during isometric, concentric, and eccentric contractions. J Appl Physiol 2001; 91: 2628-34.

[20] Duclay J, Martin A, Robbe A, Pousson M. Spinal reflex plasticity during maximal dynamic contractions after eccentric training. Med Sci Sports Exerc 2008; 40: 722-34.

[21] Amiridis IG, Martin A, Morlon B, et al. Co-activation and tension regulating phenomena during isokinetic knee extension in sedentary and highly skilled humans. Eur J Appl Physiol 1996; 73: 14956.

[22] Andersen LL, Andersen JL, Magnusson SP, Aagaard P. Neuromuscular adaptations to detraining following resistance training in previously untrained subjects. Eur J Appl Physiol 2005; 93: 511-8.

[23] Del Balso C, Cafarelli E. Adaptations in the activation of human skeletal muscle induced by short-term isometric resistance training. J Appl Physiol 2007; 103: 402-11.

[24] Higbie EJ, Cureton KJ, Warren GL, Prior BM. Effects of concentric and eccentric training on muscle strength, cross-sectional area, and neural activation. J Appl Physiol 1996; 81: 2173-81.

[25] Hortobagyi T, Hill JP, Houmard JA, Fraser DD, Lambert NJ, Israel RG. Adaptive responses to muscle lengthening and shortening in humans. J Appl Physiol 1996; 80: 765-72.

[26] Hortobagyi T, Dempsey L, Fraser D. Changes in muscle strength, muscle fibre size and myofibrillar gene expression after immobilization and retraining in humans. J Physiol 2000; 524(1): 293-304.
[27] Norrbrand L, Fluckey JD, Pozzo M, Tesch PA. Eccentric overload appears necessary to optimize skeletal muscle adaptations to chronic resistance exercise. Eur J Appl Physiol 2008; 102: 271-81.

[28] Seger JY, Arvidson B, Thorstensson A. Specific effects of eccentric and concentric training on muscle strength and morphology in humans. Eur J Appl Physiol 1998; 79: 49-57.

[29] Butterfield TA, Leonard TR, Herzog W. Differential serial sarcomere number adaptations in knee extensor muscles of rats is contraction type dependent. J Appl Physiol 2005; 99: 1352-58.

[30] Butterfield TA, Herzog W. Strain does not influence serial sarcomere number adaptations following eccentric exercise. Pflugers Arch 2006; 451: 688-700.

[31] Lynn R, Morgan DL. Decline running produces more sarcomeres in rat vastus intermedius muscle fibers than does incline running. J Appl Physiol 1994; 77: 1439-44.

[32] Lynn R, Talbot JA, Morgan DL. Differences in rat skeletal muscles after incline and decline running. J Appl Physiol 1998; 85: 98-104.

[33] Malisoux L, Francaux M, Nielens H, Theisen D. Stretch-shortening cycle exercises: an effective training paradigm to enhance power output of human single muscle fibers. J Appl Physiol 2006; 100: 771-9.

[34] Vissing K, Brink M, Lønbro S, et al. Muscle adaptations to plyometric vs. resistance training in untrained young men. J Strength Cond Res 2008; 22: 1799-810.

[35] Cronin J, Sleivert G. Challenges in understanding the influence of maximal power training on improving athletic performance. Sports Med 2005, 35: 213-34.

[36] Häkkinen K, Komi OV, Alén M, Kauhanen H. EMG, muscle fibre and force production characteristics during a 1 year training period in elite weight-lifters. Eur J Appl Physiol 1987; 56: 419-27.

[37] Fry AC. The role of resistance exercise intensity on muscle fibre adaptations. Sports Med J 2004; 34: 663-79.

(C) Per Aagaard; Licensee Bentham Open.

This is an open access article licensed under the terms of the Creative Commons Attribution Non-Commercial License (http://creativecommons.org/licenses/by-nc/3.0/) which permits unrestricted, non-commercial use, distribution and reproduction in any medium, provided the work is properly cited. 